\title{
Joint inversion of geophysical and hydrological data for improved subsurface characterization
}

\author{
Michael B. Kowalsky, Jinsong Chen and Susan S. Hubbard, Lawrence Berkeley National Lab., Berkeley, California, U.S.
}

Understanding fluid distribution and movement in the subsurface is critical for a variety of subsurface applications, such as remediation of environmental contaminants, sequestration of nuclear waste and $\mathrm{CO}_{2}$, intrusion of saline water into fresh water aquifers, and the production of oil and gas. It is well recognized that characterizing the properties that control fluids in the subsurface with the accuracy and spatial coverage needed to parameterize flow and transport models is challenging using conventional borehole data alone. Integration of conventional borehole data with more spatially extensive geophysical data (obtained from the surface, between boreholes, and from surface to boreholes) shows promise for providing quantitative information about subsurface properties and processes. Typically, estimation of subsurface properties involves a two-step procedure in which geophysical data are first inverted and then integrated with direct measurements and petrophysical relationship information to estimate hydrological parameters. However, errors inherent to geophysical data acquisition and inversion approaches and errors associated with petrophysical relationships can decrease the value of geophysical data in the estimation procedure. In this paper, we illustrate using two examples how joint inversion approaches, or simultaneous inversion of geophysical and hydrological data, offer great potential for overcoming some of these limitations.

Background. The lack of sufficient direct measurements and the ubiquity of subsurface heterogeneity typically limits our ability to accurately predict subsurface fluid migration. Conventional measurements, such as core analyses, borehole geophysical logs, and hydraulic tests, can be used to develop an understanding of hydrogeological heterogeneity in the near vicinity of the sampling boreholes. Oftentimes, interpolation between wellbores is performed to infer the spatial distribution of the measured properties, such as lithofacies. When the correlation length of the hydrogeological properties in the horizontal direction is large compared to the wellbore spacing, such interpolation can provide adequate information about hydrogeological heterogeneity for parameterizing flow models and predicting system behavior. However, more often, the wellbore spacing is much greater than the length scale of the heterogeneity, rendering inadequate characterization of the properties that control flow and transport. As a result, field-scale numerical modeling efforts often fail to accurately predict fluid migration.

From the perspective of a hydrogeological modeler, the merit of any particular geophysical data set depends on its ability to provide quantitative site-specific information about the flow and transport properties at a reasonable resolution. Integration of hydrological and geophysical data within an estimation framework offers the possibility of providing quantitative estimates of flow and transport parameters at locations unsampled by conventional borehole measurements. Several modeling studies have now shown that inclusion of hydrogeological parameters, obtained using geophysical data, greatly improves estimates of flow processes over those model results obtained using conventional characterization data alone.

The most typical approach for integrating hydrogeological and geophysical data involves a two-step hydrogeophysical procedure, whereby inversion of geophysical data is first performed to obtain estimates of geophysical attributes (e.g., radar velocity estimates are obtained through inversion of radar slowness measurements, and seismic attenuation estimates are obtained through inversion of seismic amplitude data). The geophysical attributes are considered hard data and are analyzed for correlation with direct borehole hydrogeological measurements, thereby providing the relationship between geophysical attributes and the hydrogeological parameters of interest. In the second step, the estimates of hydrological parameters are obtained using the geophysical data, petrophysical relationship, and direct borehole data through direct mapping, geostatistical, or Bayesian methods. The majority of such hydrogeophysical studies have been performed using tomographic data, for which the measurement scales of geophysical and wellbore data are often comparable, and the geophysical data coverage is acceptable. Even under these favorable conditions, application of the two-step technique for mapping hydrological parameters is limited by errors associated with geophysical data acquisition and inversion procedures, as well as inferred relationships of geophysical attributes with petrophysical properties. However, our recent research indicates that joint inversion of geophysical and hydrological data sets can reduce errors in both geophysical and hydrological parameter estimation.

In this study, we illustrate two case studies in which the geophysical and hydrological measurements were jointly inverted, effectively circumventing some of the obstacles commonly encountered during the two-step hydrogeophysical approach and taking advantage of the complementary nature of geophysical and hydrological data. Although it seems intuitive that inversion of geophysical data can benefit from inclusion of hydrogeological constraints (and vice-versa), hydrogeophysical joint inversion approaches have only recently been developed, and are rarely performed in practice. One reason for the lack of applications is the lack of effective methods for joint inversion. The first example we present in this paper focuses on improving estimates of hydrological parameters, such as permeability, relative to those obtained through conventional approaches, through joint inversion of time-lapse ground-penetrating radar (GPR) data and hydrological measurements. The second example focuses on estimating fracture zonation by joint inversion of seismic travel time data and borehole flowmeter data, where a sequential, two-step estimation procedure was not possible.

Joint inversion of time-lapse GPR travel times and borehole water content data. The use of GPR methods for mapping water distributions in the subsurface is now well established, and made possible by good correlation between the soil water content and measured dielectric constant. However, in general, GPR measurements cannot be directly related to the soil hydraulic parameters needed to make hydrological predictions in the vadose zone (such as the absolute permeability and the parameters describing the 
relative permeability and capillary pressure function). On the other hand, time-lapse GPR data likely contain information that can be indirectly related to the soil hydraulic properties, since these soil hydraulic properties influence the time- and space-varying changes in water distribution, which in turn affect GPR data.

We recently developed an approach for incorporating time-lapse GPR measurements and measurements of hydrological properties into a hydrological-geophysical joint inversion framework for estimating soil hydraulic parameter distributions. Coupling between the hydrological and GPR simulators is accomplished within the framework of iTOUGH2. Inversion is performed using a maximum a posteriori (MAP) method that utilizes concepts from the pilot point method. One of the benefits of this approach is that it directly uses GPR travel times without requiring creation of velocity tomograms, thus alleviating difficulties inherent to tomographic inversion and allowing for sparser GPR data sets, relative to those required for conventional tomography. This joint inversion method was later extended (Kowalsky et al., 2005) to account for uncertainty in the petrophysical function (water content relationship to the dielectric properties) and to increase the flexibility of GPR data characteristics (to include multiple offset data acquisition in three dimensions), allowing increased resolution and accuracy of soil hydraulic parameter estimates.

We applied this approach to data collected at the 200 East Area of the U.S. Department of Energy (DOE) Hanford site in Washington as part of an ongoing effort to develop techniques for monitoring and predicting the spread of radioactive waste and other toxic fluids present at the site. The experiment discussed here was designed in part to evaluate the effectiveness of various geophysical methods and involved the injection of water into the ground while collecting numerous hydrological and geophysical data sets, including dense sets of neutron-probe (NP)-derived water content data and GPR data (Figures 1 and 2).

Unknown parameters estimated using the joint inversion approach included log-permeability values at pilot point locations (which are used to create three-dimensional permeability distributions), porosity, a parameter of the petrophysical function (the dielectric constant of the solid component of the soil), and the water injection rates, which were not measured precisely. Joint inversion resulted in hydrological parameter distributions (Figure 3), conditioned to GPR travel times and to the measured hydrological properties, which could then be used to predict fluid flow at future times.

The accuracy of predictions for future times was evaluated through comparison with dense NP data collected at later times but not used in the inversion (Figure 4). In the first case, inversion was performed using only NP data collected in two wells at three different times (Figure 4b). In the second case, inversion was performed using GPR data collected at two times in addition to the NP data used in the first case. Compared to predictions made through inversion of a single data type (the NP data set), inclusion of GPR data in the joint inversion resulted in more accurate estimates of water content at later times. The joint inversion procedure provided information about hydrological parameters that was necessary for accurately constraining flow and transport models.

Joint inversion of crosshole seismic and borehole flowmeter data. In this example, we demonstrate another approach for combining hydrological and geophysical data. Unlike the previous example, which used time-lapse data to estimate permeability in the vadose zone, the goal of this application was to estimate fracture zonation in the saturated zone using crosshole seismic traveltime and borehole flowmeter data. For each of the pixels that comprise the tomographic planes between boreholes, the unknown variables in this case are the seismic slowness (the inverse of seismic velocity) values and hydrogeological zonation indicators obtained from borehole flowmeter data, where high hydraulic conductivity values were associated with fracture zones and lower hydraulic conductivity values were associated with competent rock. A physically based numerical model was used to relate seismic slowness to the zonation indicators. Parameters of the model were initially considered to be unknown, and were subseqeuntly estimated using a Bayesian model with Markov Chain Monte Carlo (MCMC) methods by conditioning with crosshole seismic traveltimes and the borehole flowmeter data (Chen et al., 2006)

The joint inversion approach was applied at a DOE field site located at Oak Ridge National Laboratory (ORNL) in Tennessee, USA. This study was conducted at the Field Research Center (FRC) of the DOE Environmental Remediation Science Program, where biostimulation is being tested for subsurface removal of Uranium U(VI). The saturated subsurface zone of interest at this site is characterized by shallow, dipping, and fractured saprolite. Crosshole seismic measurements and borehole flowmeter tests were collected at several monitoring wells (Figure 5) to aid in the characterization of fracture zonation that was expected to control transport at the site. The hypothesis was that lower measured velocities would be indicative of areas of less competent rock, or higher hydraulic conductivity fracture zones.

Conventional inversion of seismic travel time data yielded a tomogram showing a low velocity area indicative of a high hydraulic conductivity fracture zone (Figure 6). However, a reliable field-scale petrophysical model for connecting seismic velocity to hydraulic conductivity could not be obtained, since variations in the inverted seismic velocity are a function of azimuthal direction. The seismic velocity values at a single wellbore location, extracted from two different tomograms that intersect that borehole but that extend in different directions, show that the relative locations of the velocity 'highs' and 'lows' are similar, but their absolute values are different (Figure 7). The inability to develop a reliable petrophysical relationship at this site prohibited the application of a conventional, two-step estimation approach.

To circumvent the petrophysical problem, we converted the borehole flowmeter data into indicator logs, where each value was categorized as being in a 'fractured' or 'not fractured' region based on a hydraulic conductivity cutoff value. We then jointly inverted the indicator and crosshole seismic travel times along each traverse to obtain probability distributions indicating the likelihood of encountering a fracture zone at any given pixel (Figure 8). Qualitative comparison of the fracture zonation estimates with the field bromide tracer breakthrough data as well as with results of a Uranium biostimulation experiment conducted at the same location showed that this approach adequately estimated the fractured zonation, and that the fracture zonation influenced the biostimulation efficacy. This example illustrates how the new joint inversion approach provided quantitative estimates of properties that control transport under conditions where conventional, sequential estimation approaches were prohibitive. 


\section{Conclusions and Implications}

Our work has focused on development of joint inversion approaches that reduce errors associated with more conventional sequential inversion approaches, to provide parameter estimates beyond what are typically possible using conventional approaches, and to provide quantitative parameter estimates under conditions where conventional methods fail. Although development of joint inversion methods are in their infancy, recent work suggests that in many cases, joint inversion of hydrological and geophysical data sets can lead to an improved understanding of the parameters that control fluid migration. Although the two examples illustrated here focus on near surface characterization and monitoring, the inversion approaches developed should be applicable to a wide variety of subsurface investigations throughout an equally wide range of depths.

\section{Suggested Reading.}

"Estimating the hydraulic conductivity at the south oyster site from geophysical tomographic data using Bayesian techniques based on the normal linear regression model" by Chen et al. (Water Resour. Res., 2001). "Development of a joint hydrogeophysical inversion approach and application to a contaminated fractured aquifer" by Chen et al. (Water Resour. Res., (in press), 2006). "Geochemical characterization using geophysical data and Markov Chain Monte Carlo methods: A case study at the South Oyster Bacterial transport site in Virginia" by Chen et al. (Water Resour. Res., 2004). "Assessing the resolution-dependent utility of tomograms for geostatistics" by Day-Lewis and Lane (Geophys. Res. Lett., 2004). "iTOUGH2 User's Guide” by Finsterle (LBNL report, 1999). "Estimating flow parameter distributions using ground-penetrating radar and hydrological measurements during transient flow in the vadose zone" by Kowalsky et al. (Advances in Water Resour., 2004). "Estimation of field-scale soil hydraulic and dielectric parameters through joint inversion of GPR and hydrological data" by Kowalsky et al. (Water Resour. Res., 2005). "Modeling the field-scale relationship between dielectric constant and water content in heterogeneous system" by Moysey and Knight (Water Resour. Res., 2004). "Pre-inversion corrections and analysis of radar tomographic data" by Peterson (J. of Env. and Eng. Geophysics, 2001). "Hydrogeophysics" by Rubin and Hubbard (Springer, 2005). "An Evaluation of Conditioning Data for Solute Transport Prediction" by Scheibe and Chien (Groundwater, 2003). "Vadose Zone Transport Field Study: Soil Water Content Distributions by Neutron Moderation" by Ward et al. (Report PNNL-13795, 2000).

Acknowledgements: This work was supported by Laboratory Directed Research and Development (LDRD) funding from Berkeley Laboratory, provided by the Director, Office of Science, of the U.S. DOE under contract DE-AC02-05CH11231; and by the Environmental Remediation Science Program of the U.S. DOE Office of Science under contract DEFG07 $96 E R 14726$ 


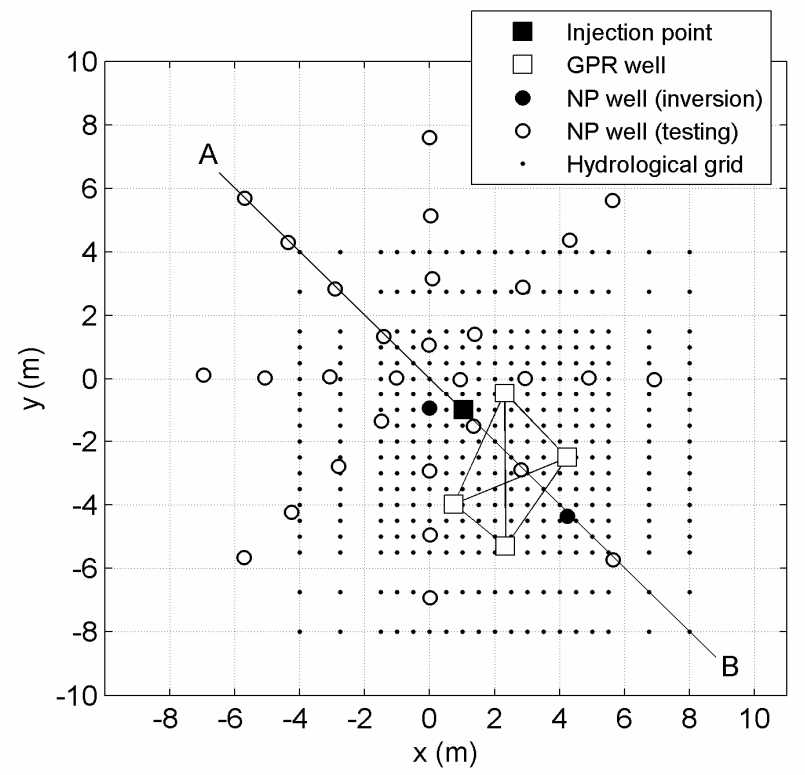

Figure 1. Measurement locations at the U.S. DOE Hanford 200 East site (Sisson and Lu, 1984) for water injection experiments conducted in FY 2000 (Ward et al., 2000). 

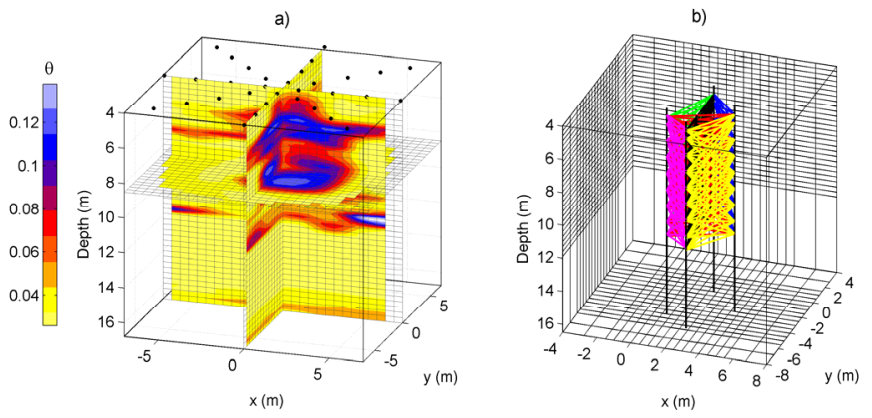

Figure 2. Time-lapse data sets collected during water injection at Hanford site include (a) water content (inferred from dense neutron-probe measurements), and (b) ground-penetrating radar. 


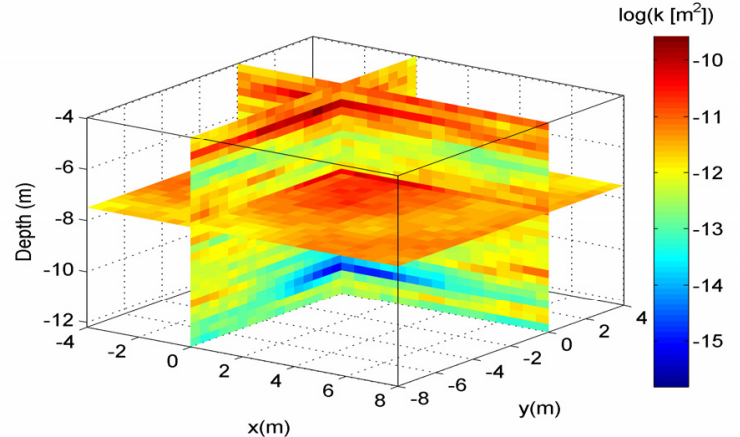

Figure 3. Realization of log permeability model estimated through joint inversion of NP and GPR data sets. 
a)

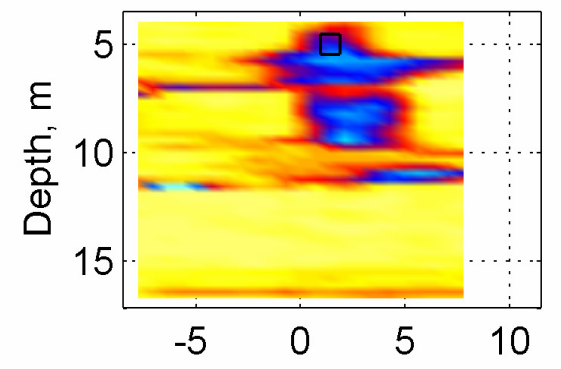

b)

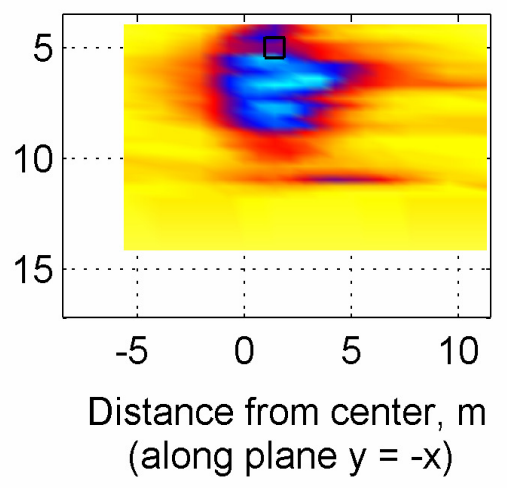

c)

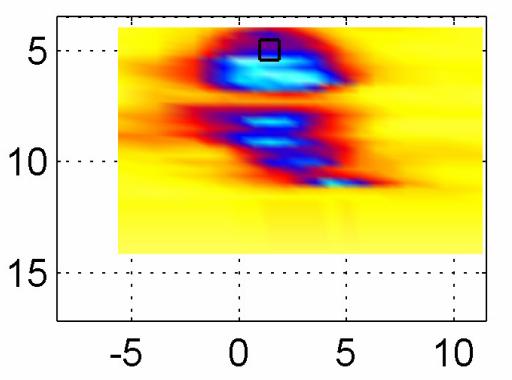

$\theta$

0.14

0.12

0.1

0.08

0.06 0.04

Figure 4. Comparison of (a) true water content with predictions made using hydrological model obtained through (b) inversion with limited NP data set (using 2 wells at three times), and (c) inversion using both GPR data sets and the limited $N P$ data sets. Two-dimensional slices (see line AB in Figure 1) from three-dimensional models are shown. 


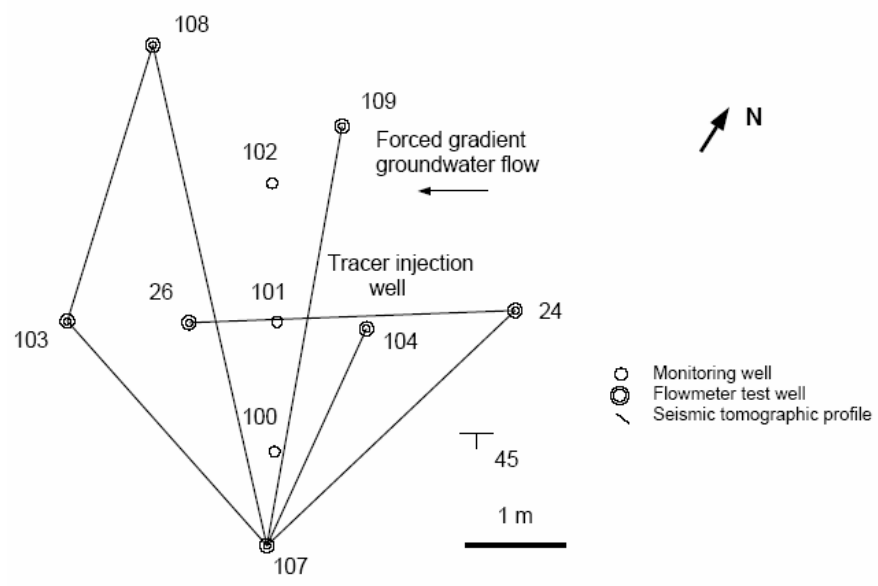

Figure 5. Geometry and locations of borehole and seismic tomographic data at the FRC Area 3 study site at Oak Ridge National Laboratory in Tennessee. 


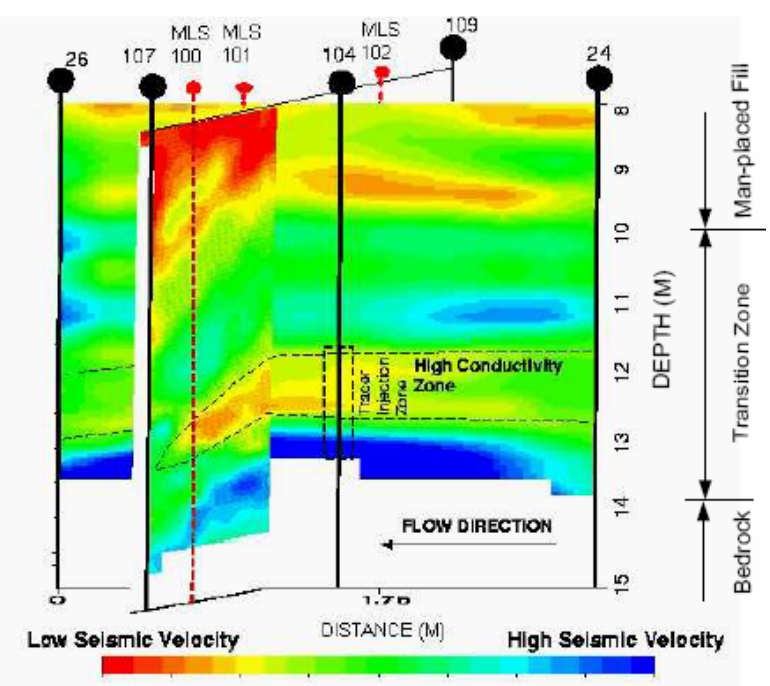

Figure 6. Conventionally inverted seismic velocity tomograms illustrating the low velocity zone that is the focus of a biostimulation experiment. 
(a)

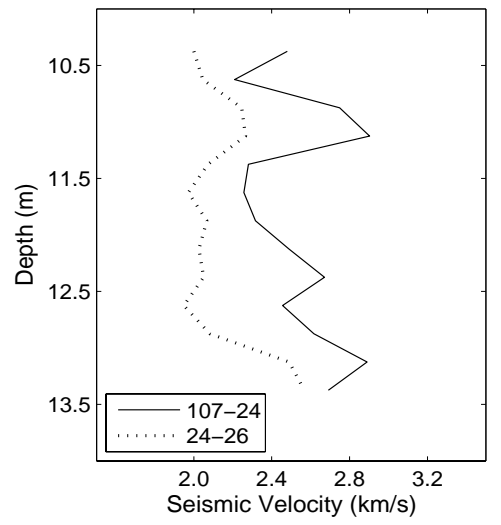

(b)

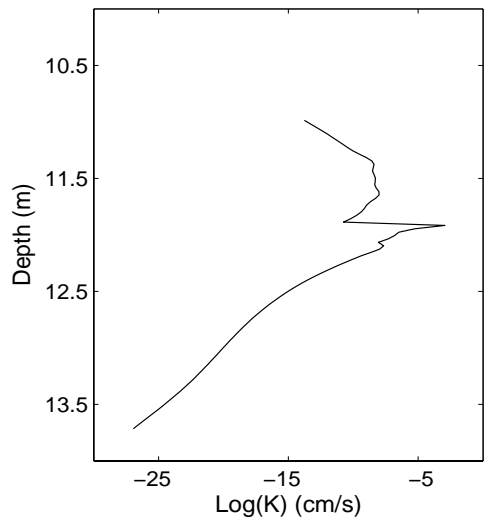

Figure 7. (a) Seismic velocity extracted from the (tomogram) transects 107-24 and 26-24 near well 24. (b) Hydraulic conductivity collected from well 24. 


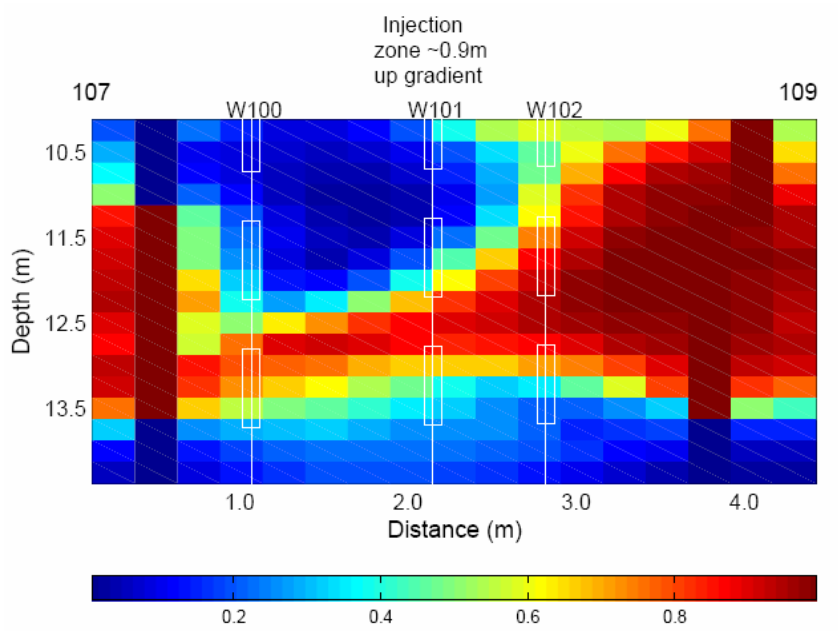

Figure 8. Probability of a pixel being in the high hydraulic conductivity fracture zone. The white boxes represent the multiple sampler intervals used during the field bromide tracer and Uranium biostimulation experiments. 